Child Health Record (PCHR). The study population was a subsample of children from ALSPAC who had height/length or weight measured both at an ALSPAC clinic and in the PCHR within two months of the ALSPAC clinic. Since ALSPAC and PCHR measurements were taken at slightly different ages, and since young children grow rapidly, comparisons needed to take account of age differences of measurements. To allow for this, comparisons were made between standard deviation scores (sds) using the 1990 growth reference. The difference between sds from clinic and PCHR measurements was assessed. If PCHR measurements are accurate, a mean difference of zero could be expected, assuming most children will not cross centiles of the reference curves in the (maximum) two months between measurements. Differences between height/length and weight measurements were assessed at age four months $(\mathrm{N}=345)$, eight months $(N=1051), 12$ months $(N=139), 18$ months $(N=649)$, 25 months $(N=183)$, and 43 months $(N=123)$. Differences were examined using summary statistics and Bland-Altman plots; predictors of the differences were explored using linear regression.

Results: Accuracy of the routinely collected measurements was generally good. For weight, all mean differences were less than 0.08 sds (e.g. mean sds differences: at 4 months 0.049 , 95\% level of agreement -1.07 to 0.97 , at 12 months 0.0025 , 95\% level of agreement -0.77 to 0.76$)$. For height/length, mean sds differences were somewhat bigger than for weight, but still generally small (e.g. mean sds differences: at 4 months $0.43,95 \%$ level of agreement -2.16 to 1.29 , at 12 months $0.16,95 \%$ level of agreement -1.32 to 1.00). The observed differences were not strongly or consistently affected by child anthropometry, sex, social class, birth weight, birth length, or maternal anthropometry.

Conclusion: Routinely collected height/length and weight data appear to have generally good accuracy, supporting their use in both clinical practice and research.

\section{PARENTAL RECOGNITION OF OVERWEIGHT IN CHILDREN AGED 6-8 YEARS: FINDINGS FROM THE GATESHEAD MILLENNIUM STUDY}

${ }^{1}$ AR Jones, ${ }^{1} \mathrm{KN}$ Parkinson, ${ }^{2} \mathrm{RF}$ Drewett, ${ }^{3} \mathrm{RM}$ Hyland, 'AJ Adamson, the Gateshead Millennium Study Core team. ${ }^{~}$ Human Nutrition Research Centre, Institute of Health and Society, Newcastle University, Newcastle upon Tyne, UK; ${ }^{2}$ Department of Psychology, Durham University, Durham, UK; ${ }^{3}$ School of Education, Durham University, Queen's Campus, Stockton on Tees, UK

\section{doi:10.1136/jech.2009.096735b}

Objectives: To investigate parental recognition of childhood overweight and explore parents' understanding of and attitudes to adiposity in childhood.

Design: Questionnaires and focus groups within a longitudinal birth cohort study.

Setting: Gateshead, UK.

Participants: 539 parents of children in the Gateshead Millennium Study, of which 27 attended 6 focus discussion groups.

Main Outcome Measures: Parental perception of their child's weight status according to actual weight status as defined by International Obesity Taskforce (IOTF) cut-offs. Outcomes from focus groups included parental awareness of childhood overweight nationally, methods used by parents to identify overweight children and grounds for not engaging with the issue.

Results: There was poor agreement between perceived child weight status and actual weight status $(k=0.297)$. More than two-thirds $(68.8 \%)$ of parents of overweight or obese children identified their child as being of "normal" weight. Of those children correctly identified as being "overweight", $66.7 \%$ were in fact obese according to IOTF criteria. Although during focus groups parents demonstrated an awareness of childhood overweight being a problem nationally, they underestimated its prevalence amongst the population. Parents tended to identify childhood overweight by using visual assessments and by comparing children within a class, school or area where only extreme cases were again categorised as "overweight". Consequently, the apparent lack of relevance of the problem to their children's school or own community, together with scepticism towards both media messages and clinical guidelines used to identify childhood overweight, commonly emerged as grounds for failing to recognise the issue. Parents also stated that diagnoses should be treated with caution as a child's body continually changes during the maturation process and phases of "chubbiness" may eventually be overcome during "growth spurts".

Conclusions: Parental recognition of both their child's overweight status and the scale of the problem nationally were poor. Parents neither use nor trust clinical measures and rely on extreme cases to identify unhealthy weight status in children. There is an urgent need to find methods to improve parental recognition of childhood overweight and obesity if parents are to play a full role in preventing this increasing public health problem.

\section{A 10-YEAR DECLINE IN SELF-RECOGNITION OF OBESITY: TRENDS IN SENSITIVITY AND SPECIFICITY FROM THREE POPULATION SURVEYS IN IRELAND}

${ }^{1} \mathrm{~F}$ Shiely, ${ }^{2} \mathrm{~K}$ Hayes, ${ }^{1} \mathrm{~J}$ Lutomski, ${ }^{1} \mathrm{~J}$ Harrington, ${ }^{3} \mathrm{H}$ McGee, ${ }^{4} \mathrm{CC}$ Kelleher, ${ }^{1} \mathrm{IJ}$ Perry. ${ }^{1}$ Department of Epidemiology and Public Health, University College Cork, Cork, UK; ${ }^{2}$ Department of Mathematics and Statistics, University of Limerick, Limerick, Ireland, ${ }^{3}$ Division of Population Health Sciences, Royal College of Surgeons in Ireland, Dublin, Ireland; " School of Public Health and Population Sciences, University College Dublin, Dublin, Ireland

\section{doi:10.1136/jech.2009.096735c}

Objectives: To examine trends in sensitivity and specificity of selfreported vs clinically measured body mass index (BMI) from three adult national lifestyle surveys over a 10 year period.

Design and Participants: Cross-sectional studies involving nationally representative samples in 1998, 2002 and 2007. Data on both self-reported and measured height and weight were available from 66 men and 142 women in 1998, 147 men and 184 women in 2002 and 909 men and 1128 women in 2007.

Setting: Interview based survey/measurement in mobile clinic or household of three representative samples in Ireland.

Main Outcome Measures: Trends in sensitivity and specificity in BMI classification of normal weight, overweight and obesity based on self-reported vs clinically measured height and weight.

Results: Sensitivity scores for the normal category improved across time but decreased for the overweight $(75.3 \% \rightarrow 67.6 \% \rightarrow 66 \%)$ and obese categories $(79.5 \% \rightarrow 64 \% \rightarrow 53.4 \%)$, demonstrating a decrease in the capacity for self-diagnosis of obesity. Simultaneously, specificity levels in the obese category remained consistently high $(100 \% \rightarrow 98 \% \rightarrow 98.2 \%)$. In all three surveys, the sensitivity for the normal BMI category was high (>94\%) indicating truly healthy subjects self-reported as healthy. Sensitivity and specificity varied by gender. Trends in measured obesity levels increased between SLÁN1998 and SLÁN2002 (21.2\%-26\%) but decreased to $24.4 \%$ in SLÁN2007. The differential between self-reported and measured obesity increased across the three surveys $(4.4 \% \rightarrow 7.9 \% \rightarrow 10 \%)$.

Conclusion: While the capacity for self-recognition of normal BMI has improved over time, increasing numbers of overweight and obese people underreport their weight. Problems of misclassification are greatest in the overweight and obese categories resulting in an underreporting of true overweight and obesity levels in the Irish population. The declining sensitivity, accompanied by rising levels of obesity in the population, suggests social norms may be at play, and has important implications for future public health interventions. 